\title{
10. DISTRIBUTION OF Au AND Pd IN BASALTS AND DIABASES IN HOLE 504B, LEGS 69 AND 140 ${ }^{1}$
}

\author{
Alexandr F. Korobeynikov ${ }^{2}$ and Nikolay N. Pertsev ${ }^{3}$
}

\begin{abstract}
Au contents have been determined in 77 samples of basalts and sheeted diabase dikes. Pd has been evaluated in 39 of the samples. The mean amount of $\mathrm{Au}$ is 3 parts per billion (ppb), fluctuating from 0.4 to $10 \mathrm{ppb}$. Au contents appear to be independent in type and intensity of alteration as well as with depth sub-bottom, although in the lower part of Hole 504B, 1900-2000 mbsf, Au contents are markedly decreased (mean: $1.1 \mathrm{ppb}$ ) and show a distinct correlation with a decrease in $\mathrm{Zn}$ contents. Pd contents vary from 2 to $360 \mathrm{ppb}$ (mean: $37 \mathrm{ppb)} \mathrm{Pd} \mathrm{is} \mathrm{higher} \mathrm{in} \mathrm{basalts} \mathrm{(53.7} \mathrm{ppb)} \mathrm{and} \mathrm{lower} \mathrm{in} \mathrm{diabase} \mathrm{dikes} \mathrm{(} 30 \mathrm{ppb}$ ), especially in moderately or strongly altered ones (12.5 ppb).
\end{abstract}

\section{INTRODUCTION}

We have undertaken studies to find out contents and distributions of gold and palladium in diabase dikes and basalts from Hole 504B. Toward this end we have used the main part of Pertsev's collection of samples from Deep Sea Drilling Project Leg 69 and Ocean Drilling Program (ODP) Leg 140 as well as the whole collection (12 samples) received by Korobeynikov from Leg 140.

To date we have made 77 determinations of Au content and 39 of Pd.

One of the purposes of the investigation is to compare the noble metal contents in the upper part of the ocean basement - that is, basalt pillow lavas and flows - with those in sheeted diabase dikes. In addition, we addressed other problems, such as dependence of the element distribution upon the depth, type, or intensity of alteration and on some geochemical features of rock units. We have compared the Au and Pd contents measured in our samples to shipboard geochemical data characterizing the petrologic units from which we obtained our samples (Cann, Langseth, Honnorez, Von Herzen, White, et al., 1983; Dick, Erzinger, Stokking, et al., 1992).

\section{ANALYTICAL METHODS}

$\mathrm{Au}$ and $\mathrm{Pd}$ contents have been determined by the polarographic and the inversion-voltampermetric methods (Kolpakova et al., 1970, 1991) in the Tomsk Polytechnic University, analyst G.A. Novikova.

Au content was estimated from charges of $1 \mathrm{~g}$ by the method of film polarography with build-up on the graphite electrode. The sensitivity of the method is $0.01 \mathrm{ppb}$; reproducibility is $90 \%$ with control by internal and external standards. Differences of results in parallel runs are usually within the $12 \%-40 \%$ level and are mostly due to the distribution dispersion of gold in a sample. Extraction of $\mathrm{Au}$ was conducted twice by the ethyl- or diethyl-ether from water solutions of $\mathrm{HBr}$.

The Pd contents have been determined by the method of inversion volt-ampermetry with the carbon-past electrode. The method permits us to evaluate Pt-group element contents in charges $1-50 \mathrm{~g}$ in compositional intervals from $1 \mathrm{ppb}$ to several percent (by weight). The reproducibility of the method reaches $80 \%-90 \%$ (Kolpakova et al., 1986).

\footnotetext{
'Erzinger, J., Becker, K., Dick, H.J.B., and Stokking, L.B. (Eds.), 1995. Proc. ODP, Sci. Results, 137/140: College Station, TX (Ocean Drilling Program).

${ }^{2}$ Tomsk Polytechnic University, Tomsk, 634004, Russia.

${ }^{3}$ Institute of Geology of Ore Deposits, Petrography, Mineralogy, and Geochemistry, Russian Academy of Sciences, Moscow, 109017, Russia.
}

\section{RESULTS AND DISCUSSION}

The Au and Pd contents are presented in Table 1 and Figure 1, along with sample depths, alteration peculiarities, and geochemical features of corresponding petrological units. Both basalts and diabase dikes have moderately low mean contents of gold ( 3.3 and $2.7 \mathrm{ppb}$, respectively). The distribution of the gold in the samples, however, is not uniform. In basalts, relatively low values (1-0.5 ppb) were found in samples from 442, 474, 482, 483, and 485 mbsf. Higher concentrations, 4.5-6 ppb, were noted at 283, 287, 430, 463, 477, and 484 mbsf. We have detected no differences in Au content between pillow edges and their interior. The samples of basalts showing noticeable oxidative alteration $(n=13)$ give almost the same mean for gold $(3.2 \mathrm{ppb})$ as the samples $(n=17)$ with only reductive or neutral oxidation (3.3 ppb).

The intensity of basalt alteration does not have a detectable influence on the Au distribution. Eleven mostly unaltered basalt samples have $3.2 \mathrm{ppb}$, whereas 19 specimens that are moderately or strongly replaced by secondary minerals give $3.3 \mathrm{ppb}$.

In sheeted dikes recovered during Legs 137 and 140, three intervals are characterized by different mean amounts of gold, as shown in Table 2. Here, too, alteration has no significant influence on gold contents in spite of the change in types of secondary mineral assemblages from clay minerals in the upper (basalt) part to greenschist minerals in the lower (dike) part. The weakly altered rocks $(n=23)$ have $2.5 \mathrm{ppb}$, and moderately or strongly replaced ones $(n=24)$ contain $2.9 \mathrm{ppb}$. The highest Au concentration (10 ppb) appears to be associated with a higher than usual pyrite content in the sample (Sample 140-504B-202R-1, Piece 1).

The considerably lower Au contents in the lower part of the hole (mean $1.1 \mathrm{ppb}$ ) seem to correlate with the noticeable decrease in $\mathrm{Zn}$ contents found by the shipboard investigation (Dick, Erzinger, Stokking, et al., 1992), possibly due to increased leaching of metals in that part of the hydrothermal cell.

On the whole, the distribution of gold recovered in Hole 504B from ocean basement generally follows a log-normal low: $\mathrm{A} / \mathrm{S}_{\mathrm{A}}$ (ratio of skewness to variance of skewness) $=3.38$ and $E / S_{E}$ (ratio of excess to variance of excess $)=4.94$. The mean Au content ( $3 \mathrm{ppb}$ ) compares well with basalts and diabases of other regions (Korobeynikov, 1988).

The mean amount of $\mathrm{Pd}$ in our samples is $37 \mathrm{ppb}(n=39)$. $\mathrm{Pd}$ distribution in the basement recovered in Hole 504B, however, is not uniform. Pd amounts in basalts range from 4 to $120 \mathrm{ppb}$ (Table 1) with a mean of $53.7 \mathrm{ppb}(n=10)$. In diabase dikes, Pd content ranges from 2 to $360 \mathrm{ppb}(n=28)$ with a mean of $30 \mathrm{ppb}$. The reason for the especially high Pd concentration ( $360 \mathrm{ppb})$ in a sample of rather fresh diabase (Sample 137-504B-173R-2, 14-16 cm, Piece 2) is not yet clear. All samples of diabase $(n=28)$ display a mean of $30 \mathrm{ppb}$. If we exclude the specimen with especially high Pd $(n=27)$, we get only $17.7 \mathrm{ppb}$. Weakly altered or fresh diabases show $56.8 \mathrm{ppb}(n=11)$ or 
Table 1. Au and Pd contents and some geochemical characteristics of samples from Hole 504B.

\begin{tabular}{|c|c|c|c|c|c|c|c|c|c|c|c|c|}
\hline \multirow[b]{2}{*}{$\begin{array}{l}\text { Core, section, } \\
\text { interval (cm) }\end{array}$} & \multirow[b]{2}{*}{ Piece } & & & Alter: & & & & & & & & \\
\hline & & Unit & $\begin{array}{l}\text { Depth } \\
\text { (mbsf) }\end{array}$ & Intensity & Type & $\begin{array}{c}\mathrm{Au} \\
(\mathrm{ppb})\end{array}$ & $\begin{array}{c}\mathrm{Pd} \\
(\mathrm{ppb})\end{array}$ & $\mathrm{Mg}^{\circ}$ & $\begin{array}{l}\mathrm{CaO} / \mathrm{Na}_{2} \mathrm{O} \\
\text { (wt) }\end{array}$ & $\begin{array}{l}\mathrm{Zr} / \mathrm{Y} \\
(\mathrm{wt})\end{array}$ & $\begin{array}{c}\mathrm{TiO}_{2} / \mathrm{Cr} \\
\text { (wt) }\end{array}$ & $\underset{(\mathrm{ppm})}{\mathrm{Zn}}$ \\
\hline Leg 69 . Hole 504B & & & & & & & & & & & & \\
\hline $4-2,2-17$ & 291 & $2 \mathrm{~A}$ & 282 & $\mathrm{~S}$ & $\mathrm{R}$ & 4.2 & & 0.63 & 7.06 & 1.78 & 18.1 & 65 \\
\hline $4-2,18-20$ & 292 & $2 \mathrm{~A}$ & 284 & W & Ox & 5.4 & & 0.65 & 6.12 & 2.26 & 20.6 & 53 \\
\hline $4-3,94-96$ & 320 & $2 \mathrm{~A}$ & 285 & W & $\mathrm{R}$ & 3.3 & & & & & 20.6 & 62 \\
\hline $4-4,82-85$ & 336 & $2 \mathrm{~A}$ & 287 & $\mathrm{M}$ & $\hat{R}$ & 4.6 & & 0.62 & 6.98 & 2.18 & 20.6 & 6.8 \\
\hline $4-5,75-77$ & 352 & $2 \mathrm{~A}$ & 289 & $\mathrm{M}$ & Ox & 4.2 & 60 & 0.62 & 7.74 & 2.04 & 20.6 & 66 \\
\hline $6-2,95-97$ & 422 & $2 \mathrm{~B}$ & 306.7 & M & Ox & 4.0 & & 0.66 & 6.29 & 2.26 & 24.4 & 81.7 \\
\hline $6-2,120-122$ & 424 & $2 \mathrm{~B}$ & 307.1 & $\mathrm{M}$ & Ox & 3.0 & 4 & 0.65 & 6.15 & 2.17 & 22.1 & 81 \\
\hline $7-2,103-105$ & 464 & $2 \mathrm{C}$ & 312 & M & Ox & 3.6 & 50 & 0.63 & 5.75 & 2.04 & 23.0 & 67 \\
\hline $8-1.87-90$ & 524 & $2 \mathrm{D}$ & 317 & $\mathrm{M}$ & Ox & 3.1 & 20 & 0.67 & 5.90 & 2.09 & 22.8 & 69 \\
\hline $15-1.72-75$ & 898 & $3 \mathrm{C}$ & 379 & M & $\mathrm{R}$ & 2.7 & 6 & 0.64 & 6.73 & 1.89 & 32.6 & 60 \\
\hline $15-4,65-67$ & 934 & $3 \mathrm{C}$ & 381 & w & $\hat{\mathrm{R}}$ & 3.6 & & 0.65 & 6.74 & 1.89 & & \\
\hline $16-2,27-29$ & 976 & $3 \mathrm{C}$ & 385 & M & Ox & 2.0 & & 0.63 & 6.73 & 2.00 & 27.2 & 85 \\
\hline $16-4,52-54$ & 1008 & 4 & 390 & $\mathrm{M}$ & Ox & 3.6 & 120 & 0.67 & 5.72 & 2.40 & 27.2 & 115 \\
\hline $17-2,72-75$ & 1062 & 4 & 395 & W & $\mathrm{R}$ & 3.3 & 100 & 0.67 & 6.02 & 2.37 & 19.1 & 73 \\
\hline $19-2,108-112$ & 1149 & 6 & 410 & $\mathrm{~s}$ & Ox & 2.5 & & 0.61 & 6.29 & 1.97 & 19.4 & 64 \\
\hline $21-2,47-54$ & 1203 & 9 & 422 & W & $\mathrm{R}$ & 3.9 & & 0.66 & 7.41 & 1.64 & 18.0 & 70 \\
\hline $21-2,115-117$ & 1214 & 10 & 423 & W & $\ddot{R}$ & 3.4 & 91 & 0.65 & 6.70 & 1.68 & 22.6 & \\
\hline $21-3,146-148$ & 1236 & II & 425 & $M$ & Ox & 3.3 & & 0.68 & 6.24 & 1.91 & 19.5 & 62 \\
\hline $21-4,98-102$ & 1251 & 14 & 429 & W & $\mathrm{R}$ & 3.0 & & 0.66 & 7.29 & 1.64 & 18.0 & 61 \\
\hline $21-5,8-11$ & 1259 & 14 & 430 & W & $\ddot{R}$ & 6.0 & & 0.66 & 7.29 & 1.64 & 18.0 & 61 \\
\hline $23-1,115-117$ & 1326 & 16 & 447 & $\mathrm{M}$ & Ox & 3.0 & & 0.65 & 6.82 & 1.72 & 26.8 & 71 \\
\hline $24-1,88-90$ & 1343 & 16 & 449 & $\mathrm{M}$ & Ox & 3.0 & & 0.65 & 6.82 & 1.72 & 21.0 & 56 \\
\hline $25-2,108-111$ & 1436 & 17 & 462 & W & Ox & 0.9 & & 0.64 & 6.20 & 2.07 & 29.2 & 83 \\
\hline $26-1,3-6$ & 1447 & 17 & 463 & M & R & 4.8 & & 0.66 & 5.65 & 1.72 & 30.5 & 69 \\
\hline $27-2,60-63$ & 1485 & 17 & 474 & W & $\hat{R}$ & 0.9 & & 0.63 & 4.91 & 2.36 & 29.2 & 83 \\
\hline $28-1,30-36$ & 1490 & 18 & 477 & $\mathrm{~s}$ & $\hat{R}$ & 4.9 & & 0.65 & 6.81 & 1.42 & 34.2 & 71 \\
\hline $28-2,50-58$ & 1508 & 19 & 481 & s & $\hat{R}$ & 5.2 & & 0.61 & 5.65 & 2.45 & 24.6 & 68 \\
\hline $28-3,87-89$ & 1533 & 19 & 482 & W & $\hat{R}$ & 1.0 & 4 & 0.65 & 5.71 & 1.89 & 31.0 & 151 \\
\hline $28-4,12-15$ & 1541 & 19 & 483 & M & $\ddot{R}$ & 0.7 & 120 & 0.65 & $5.7 i$ & 1.89 & 31.0 & 76 \\
\hline $29-1,21-23$ & 1561 & 20 & 485 & s & $\mathrm{R}$ & 0.5 & 16 & 0.63 & 6.75 & 2.05 & 31.2 & 76 \\
\hline Leg 137 , Hole $504 \mathrm{~B}$ & & & & & & & & & & & & \\
\hline $173 \mathrm{R}-2,14-16$ & 2 & 194 & 1576 & w & $\mathrm{R}$ & 1.5 & 360 & 0.62 & 9.91 & 1.82 & 36.8 & \\
\hline $176 \mathrm{R}-1,74-76$ & 14 & $\mathrm{x}$ & 1596 & W & $\mathrm{R}$ & 2.3 & 36 & & & & & \\
\hline Leg 140, Hole $504 \mathrm{~B}$ & & & & & & & & & & & & \\
\hline 186R-1. 103-105 & $14 \mathrm{~B}$ & 213 & 1630 & $s$ & $\mathbf{R}$ & 3.2 & 3 & 0.67 & 8.08 & 2.29 & 26.3 & 43.2 \\
\hline $189 \mathrm{R}-1,132-135$ & 26 & 218 & 1652 & W & R & 3.6 & 12 & 0.61 & 7.26 & 1.83 & 41.0 & 63.7 \\
\hline $19 \mid R-1,91-93$ & 13 & 218 & 1670 & w & $\ddot{R}$ & 2.7 & & 0.66 & 7.24 & 1.76 & 18.9 & 54.4 \\
\hline $192 \mathrm{R}-1,34-36$ & 10 & 218 & 1674 & M & $\hat{R}$ & 1.0 & & 0.62 & 6.66 & 1.83 & 35.5 & 70.7 \\
\hline $194 \mathrm{R}-1,50-52$ & 9 & 220 & 1685 & W & $\hat{R}$ & 2.0 & & 0.65 & 7.25 & 1.76 & 23.8 & 57.6 \\
\hline $197 \mathrm{R}-1,108-111$ & 26 & 222 & 1704 & M & $\ddot{R}$ & 6.0 & 20 & 0.67 & 7.38 & 1.80 & 21.7 & 56.2 \\
\hline $197 \mathrm{R}-2,14-16$ & 2 & 223 & 1712 & w & $\hat{R}$ & 1.2 & & 0.67 & 7.13 & 1.74 & 21.3 & 56.5 \\
\hline $200 \mathrm{R}-1,116-118$ & 20 & 227 & 1730 & w & $\mathrm{K}$ & 4.2 & 36 & 0.69 & 7.25 & 2.04 & 16.8 & 52.6 \\
\hline $200 \mathrm{R}-1,128-130$ & 21 & 227 & 1731 & W & $\mathrm{R}$ & 3.2 & 66 & 0.69 & 7.25 & 2.04 & 16.8 & 52.6 \\
\hline $200 \mathrm{R}-2,79-81$ & 10 & 227 & 1732 & w & $\begin{array}{l}K \\
R\end{array}$ & 3.0 & 00 & 0.70 & 7.35 & 2.03 & 16.8 & 49.6 \\
\hline 200R-2, 95-97 & 12 & 227 & 1733 & w & $\hat{R}$ & 4.8 & 36 & 0.70 & 7.35 & 2.03 & 16.8 & 49.6 \\
\hline $200 \mathrm{R}-3,90-92$ & 14 & 227 & 1734 & $\mathrm{w}$ & $\hat{R}$ & 2.2 & 20 & 0.69 & 7.39 & 2.06 & 18.6 & 64.3 \\
\hline $200 R-4,50-53$ & 13 & 227 & 1735 & $\ddot{M}$ & $\hat{R}$ & 4.3 & 7 & 0.63 & 6.32 & 2.07 & 22.7 & 62.0 \\
\hline $202 \mathrm{R}-1,1-4$ & $i$ & 229 & 1747 & $\mathrm{~s}$ & $\begin{array}{l}K \\
R\end{array}$ & 10.0 & 10 & 0.66 & 7.25 & 1.76 & 22.2 & 53.3 \\
\hline $202 \mathrm{R}-1,16-18$ & 4 & 229 & 1748 & w & $\hat{R}$ & 1.5 & & 0.66 & 7.25 & 1.76 & 22.2 & 53.3 \\
\hline $203 R-1,57-59$ & 15 & 232 & 1756 & $\mathrm{M}$ & $\hat{R}$ & 2.5 & 6 & 0.67 & 7.53 & 2.37 & 25.5 & 37,0 \\
\hline $204 \mathrm{R}-1,20-23$ & 5 & 232 & 1757 & $\mathrm{~S}$ & $\hat{R}$ & 2.7 & 5 & 0.67 & 7.58 & 2.34 & 24.5 & 46.8 \\
\hline $204 \mathrm{R}-1.44-46$ & 11 & 232 & 1757.5 & $\mathrm{M}$ & $\hat{R}$ & 2.0 & 10 & 0.67 & 7.58 & 2.34 & 24.5 & 46.8 \\
\hline $205 \mathrm{R}-1,35-37$ & 6 & 232 & 1759 & $\mathrm{M}$ & $\hat{\mathrm{R}}$ & 2.7 & & 0.68 & 8.00 & 2.35 & 26.0 & 56.5 \\
\hline $205 \mathrm{R}-1,40-43$ & 7 & 232 & 1759.2 & W & Ox & 4.7 & 4 & 0.68 & 8.00 & 2.35 & 26.0 & 56.5 \\
\hline $206 \mathrm{R}-1,32-34$ & 8 & 235 & 1764 & W & $\mathrm{R}$ & 1.6 & & 0.68 & 7.52 & 1.98 & 17.0 & 51.5 \\
\hline $208 \mathrm{R}-1.57-59$ & 14 & 239 & 1780 & w & $\hat{R}$ & 1.0 & & 0.66 & 7.57 & 1.92 & 19.1 & 57.6 \\
\hline $209 \mathrm{R}-1,30-31$ & 5 & 240 & 1788 & $\mathrm{M}$ & $\hat{R}$ & 2.7 & 2 & 0.64 & 6.34 & 1.76 & 9.3 & 60.7 \\
\hline $209 \mathrm{R}-1,42-44$ & 6 & 240 & 1788.2 & $\mathrm{~s}$ & $\hat{R}$ & 1.0 & 5 & 0.64 & 6.34 & 1.76 & 9.3 & 61.0 \\
\hline $209 \mathrm{R}-2,127-130$ & 20 & 240 & 1790 & s & $\hat{R}$ & $\begin{array}{l}3.0 \\
3.9\end{array}$ & 12 & 0.69 & 7.42 & 1.91 & 15.8 & 43.8 \\
\hline $21 I R-1.54-56$ & 12 & 241 & 1800 & s & $\mathrm{K}$ & 3.4 & & 0.66 & 7.08 & 1.75 & 21.6 & 46.9 \\
\hline $212 \mathrm{R}-1.55-57$ & 16 & 241 & 1807 & W & $\hat{R}$ & 2.7 & & 0.66 & 7.15 & 1.75 & 21.4 & 48.8 \\
\hline $213 \mathrm{R}-1,10-13$ & 3 & 242 & 1813 & $\mathrm{M}$ & $\hat{R}$ & 4.5 & 7 & 0.60 & 6.68 & 1.96 & 67.6 & 36.8 \\
\hline $213 R-1,100-102$ & 24 & 243 & 1818 & W & $\hat{R}$ & 4.0 & & 0.62 & 6.30 & 1.96 & 39.2 & 68.9 \\
\hline $214 \mathrm{R}-1,18-21$ & 4 & 244 & 1819 & $\mathrm{~s}$ & $\hat{R}$ & 3.2 & 60 & 0.68 & 7.00 & 1.75 & 11.2 & 39.3 \\
\hline $214 \mathrm{R}-2,23-25$ & 6 & 244 & 1821 & $\mathrm{~s}$ & $\hat{R}$ & 2.7 & 17 & 0.71 & 7.08 & 1.72 & 18.0 & 34.6 \\
\hline $215 R-1,53-55$ & 14 & 244 & 1825 & W & $\hat{R}$ & 1.7 & 12 & 0.66 & 7.27 & 1.76 & 21.4 & 43.9 \\
\hline $219 \mathrm{R}-1,13-15$ & 4 & 250 & 1865 & $\mathrm{M}$ & $\hat{R}$ & 2.5 & 11 & & & & & \\
\hline $220 \mathrm{R}-1,10-13$ & 3 & 252 & 1866 & W & $\mathrm{R}$ & 4.2 & 13 & 0.66 & 7.11 & 1.78 & 20.9 & 55.9 \\
\hline 22 IR-1, $26-28$ & 8 & 254 & 1879 & $\mathrm{M}$ & $\ddot{R}$ & 4.0 & 28 & 0.67 & 8.26 & 2.49 & 40.7 & 35.9 \\
\hline $222 \mathrm{R}-1,19-21$ & iD & 254 & 1885 & $\mathrm{M}$ & $\mathrm{R}$ & 2.9 & & 0.63 & $\begin{array}{l}6.29 \\
6.29\end{array}$ & & & \\
\hline $226 \mathrm{R}-1,45-48$ & 10 & 260 & 1922 & $\mathrm{M}$ & $\mathrm{R}$ & 0.9 & 3 & 0.67 & 7.22 & 1.79 & 22.0 & 39.3 \\
\hline $226 \mathrm{R}-2,17-20$ & 2 & 260 & 1924 & $\mathrm{M}$ & R & 2.0 & & 0.66 & 7.01 & 1.83 & 24.5 & 39.7 \\
\hline $227 \mathrm{R}-1.59-61$ & $8 \mathrm{~A}$ & 260 & 1930 & W & $\mathrm{R}$ & 0.5 & & 0.67 & 7.17 & 1.80 & 20.9 & 36.9 \\
\hline $228 \mathrm{R}-1.60-62$ & 14 & 262 & 1942 & w & $\mathrm{K}$ & 0.9 & & 0.68 & 6.85 & 1.77 & 21.3 & 49.2 \\
\hline $229 \mathrm{R}-\mathrm{I}, 14-16$ & 5 & 264 & 1950 & w & $\hat{R}$ & 0.3 & & & & & & \\
\hline $230 \mathrm{R}-2, \mathrm{I}-3$ & IA & 265 & 1953 & $\mathrm{M}$ & $\hat{R}$ & 0.4 & & 0.67 & 7.59 & 1.97 & & 22.9 \\
\hline $232 \mathrm{R}-1,12-14$ & 3 & 265 & 1958 & $\mathrm{M}$ & $\hat{R}$ & 1.3 & 7 & & & & & 23.0 \\
\hline $235 \mathrm{R}-1,8-10$ & 3 & 267 & 1980 & W & $\hat{R}$ & 10 & & & & & & \\
\hline $237 \mathrm{R}-1,3-5$ & 2 & 269 & 1990 & W & R & 0.6 & 30 & 0.65 & 6.89 & 1.90 & & 35.9 \\
\hline
\end{tabular}

Notes: $\mathrm{Mg}^{\prime \prime}=$ Molar ratio $\mathrm{MgO} /(\mathrm{MgO}+\mathrm{FeO}) ; \mathrm{FeO}$ is considered as $0.9^{*} \mathrm{Fe}_{\text {total }}$. Alteration intensity: $\mathrm{M}=$ moderate, $\mathrm{S}=$ strong, $\mathrm{W}=$ weak. Alteration type: $\mathrm{Ox}=$ oxidized, $\mathrm{R}=$ reduced or neutral. 


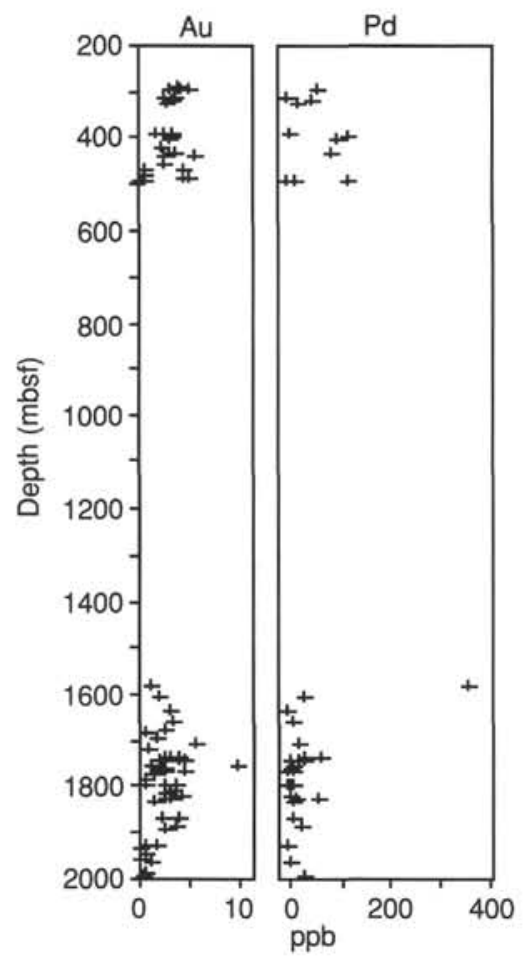

Figure 1. Au and Pd distribution in studied intervals of Hole 504B.

$26.5 \mathrm{ppb}(n=10)$, respectively. Strongly and moderately altered diabases demonstrate markedly lower Pd content of $12.5 \mathrm{ppb}(n=17)$.

The correlation analysis among Au content, depth (D), $\mathrm{Mg}^{*}$, $\mathrm{CaO} / \mathrm{Na}_{2} \mathrm{O}, \mathrm{Zr} / \mathrm{Y}, \mathrm{TiO}_{2} / \mathrm{Cr}$, and $\mathrm{Zn}$ in the rocks of the basement studied (Table 3) demonstrates the absence of stable correlations between $\mathrm{Au}$ contents and the other parameters. A very weak positive correlation seems to exist between $\mathrm{Au}$ and $\mathrm{TiO}_{2} / \mathrm{Cr}: \mathrm{r}_{\mathrm{Au}-\mathrm{TiO}, / \mathrm{Cr}}=+0.03 . \mathrm{D}, \mathrm{Zn}$, and $\mathrm{Mg}^{*}$ show unstable negative relations with Au contents: $\mathrm{r}_{\mathrm{Au}-\mathrm{D}}=$ $-0.114 ; \mathrm{r}_{\mathrm{Au}-\mathrm{Zn}}=-0.102 ; \mathrm{r}_{\mathrm{Au}-\mathrm{Mg}}{ }^{*}=-0.016$. There is a negative correlation between $\mathrm{Mg}^{*}$ and $\mathrm{TiO}_{2} / \mathrm{Cr}(\mathrm{r}=-0.478)$.

Factor analysis of Au content and the other characteristics shows positive values for $\mathrm{Au}(0.935)$ and $\mathrm{Zr} / \mathrm{Y}(0.254)$.

\section{SUMMARY}

Au contents in the rocks studied range from 0.4 to $10 \mathrm{ppb}$ and demonstrate a rather stable mean of about 3 ppb in much the same way as analogous rocks of other regions.

The data on Au distribution allow us to suggest that Au contents in basalts and diabase dikes of Hole 504B do not depend on depth or on type and intensity of alteration, as the $\mathrm{Mg}^{*}$ and $\mathrm{TiO}_{2} / \mathrm{Cr}$ values of the rocks have only weak tendencies toward negative and positive correlations with gold.

Relatively higher values of Au content (5-10 ppb) appear to be induced by higher secondary pyrite concentrations.
Table 2. Mean amounts of Au in sheeted dikes from Legs 137/140.

\begin{tabular}{cccc}
\hline $\begin{array}{c}\text { Depth } \\
\text { (mbsf) }\end{array}$ & $n$ & $\begin{array}{c}\text { Mean } \\
(\mathrm{ppb})\end{array}$ & $\begin{array}{c}\text { Standard } \\
\text { deviation }\end{array}$ \\
\hline $1576-1712$ & 9 & 2.6 & 1.46 \\
$1730-1879$ & 29 & 3.2 & 1.69 \\
$1885-1990$ & 10 & 1.1 & 0.77 \\
\hline
\end{tabular}

The low Au contents in the lower part of Hole 504B drilled during Leg 140 (1900-2000 mbsf) seem to be paragenetically connected with low $\mathrm{Zn}$ contents there, possibly due to more intense leaching in that part of the convective hydrothermal system.

Pd contents generally are much higher (mean $36 \mathrm{ppb}$ ). Their values range between 2 and $360 \mathrm{ppb}$. Higher concentration of Pd in basalts and much lower concentrations in altered diabases seem to have developed because of greenschist alteration of the diabases.

\section{ACKNOWLEDGMENTS}

The authors are indebted to ODP for the samples provided for this investigation. The work has been performed with the support of Russian Foundation of Fundamental Investigations (Program 93-05-9331) and of Russian State Scientific Program "The World Ocean." We are thankful to G.A. Novikova, N.A. Kolpakova, and Z.S. Mikhaylova (Tomsk Polytechnic University) for analytical help. We are grateful to the reviewers for valuable remarks and language corrections.

\section{REFERENCES}

Cann, J.R., Langseth, M.G., Honnorez, J., Von Herzen, R.P., White, S.M., et al., 1983. Init. Repts. DSDP, 69: Washington (U.S. Govt. Printing Office). Dick, H.J.B., Erzinger, J., Stokking, L.B., et al., 1992. Proc. ODP, Init. Repts., 140: College Station, TX (Ocean Drilling Program).

Kolpakova, N.A., Shifris, B.S., Shvets, L.A., and Kropotkina, S.V., 1991. Determination of PGE and Au by the method of inversion volt ampermetry. J. Anal. Chem., 46:1910-1913.

Kolpakova, N.A., Shifris, B.S., Shvets, L.A., and Kurilenko, N.V., 1986. Comparative potentialities of PGE determinations by the IV method on graphite and $\mathrm{Hg}$-graphite electrodes. 13th All Union Chernyaevsk Meet. on Chem., Anal., and Technol. of PGE. (Abstract)

Kolpakova, N.A., Stromberg, A.G., Kaplin, A.A., and Belousova, N.I., 1970. On the outlook for geochemistry of the new high-sensible method API. Trans. Tomsk Polytech. Inst., 239:441-445.

Korobeynikov, A.F., 1988. Gold in volcanic rocks of various composition and ages. Geochemistry, 11:1618-1626.

Abbreviations for names of organizations and publications in ODP reference lists follow
the style given in Chemical Abstracts Service Source Index (published by American Chemical Society).

Date of initial receipt: 23 March 1993

Date of acceptance: 20 October 1993

Ms 137/140SR-011 
Table 3. Statistical analysis of the variables from Table 1.

\begin{tabular}{|c|c|c|c|c|c|c|c|c|c|c|c|c|c|}
\hline \multirow[b]{2}{*}{$\begin{array}{l}\text { Depth } \\
\text { (mbsf) }\end{array}$} & \multicolumn{6}{|c|}{ Mean of each variable } & \multirow[b]{2}{*}{$\begin{array}{l}\text { Depth } \\
\text { (mbsf) }\end{array}$} & \multicolumn{6}{|c|}{ Mean of each variable } \\
\hline & $\mathrm{Mg}^{*}$ & $\mathrm{CaO} / \mathrm{Na}_{2} \mathrm{O}$ & $\mathrm{Zr} / \mathrm{Y}$ & $\mathrm{Au}$ & $\mathrm{TiO}_{2} / \mathrm{Cr}$ & $\mathrm{Zn}$ & & $\mathrm{Mg}^{*}$ & $\mathrm{CaO} / \mathrm{Na}_{2} \mathrm{O}$ & $\mathrm{Zr} / \mathrm{Y}$ & $\mathrm{Au}$ & $\mathrm{TiO}_{2} / \mathrm{Cr}$ & $\mathrm{Zn}$ \\
\hline 282 & 0.63 & 7.06 & 1.78 & 4.2 & 18.1 & 65 & 1759.2 & 0.68 & 8.00 & 2.35 & 4.7 & 26.0 & 56.5 \\
\hline 283 & 0.65 & 6.12 & 2.26 & 5.4 & 20.6 & 53 & 1754 & 0.68 & 7.52 & 1.98 & 1.6 & 17.0 & 51.5 \\
\hline 287 & 0.62 & 6.98 & 2.18 & 4.6 & 20.6 & 6.8 & 1780 & 0.66 & 7.57 & 1.92 & 1.0 & 19.1 & 57.6 \\
\hline 289 & 0.62 & 7.74 & 2.04 & 4.2 & 20.6 & 66 & 1788 & 0.64 & 6.34 & 1.76 & 2.7 & 9.3 & 60.7 \\
\hline 306.7 & 0.66 & 6.29 & 2.26 & 4.0 & 24.4 & 81.7 & 1788.2 & 0.64 & 6.34 & 1.76 & 1.0 & 9.3 & 61.0 \\
\hline 307.1 & 0.65 & 6.15 & 2.17 & 3.0 & 22.1 & 81 & 1790 & 0.69 & 7.42 & 1.91 & 3.9 & 15.8 & 43.8 \\
\hline 312 & 0.63 & 5.75 & 2.04 & 3.6 & 23.0 & 67 & 1800 & 0.66 & 7.08 & 1.75 & 3.4 & 21.6 & 46.9 \\
\hline 317 & 0.67 & 5.90 & 2.09 & 3.1 & 22.8 & 69 & 1807 & 0.66 & 7.15 & 1.75 & 2.7 & 21.4 & 48.8 \\
\hline 379 & 0.64 & 6.73 & 1.89 & 2.7 & 32.6 & 60 & 1813 & 0.60 & 6.68 & 1.96 & 4.5 & 67.6 & 36.8 \\
\hline 385 & 0.63 & 6.73 & 2.00 & 2.0 & 27.2 & 85 & 1818 & 0.62 & 6.30 & 1.96 & 4.0 & 39.2 & 68.9 \\
\hline 390 & 0.67 & 5.72 & 2.40 & 3.6 & 27.2 & 115 & 1819 & 0.68 & 7.00 & 1.75 & 3.2 & 11.2 & 39.3 \\
\hline 395 & 0.67 & 6.02 & 2.37 & 3.3 & 19.1 & 73 & 1821 & 0.71 & 7.08 & 1.72 & 2.7 & 18.0 & 34.6 \\
\hline 410 & 0.61 & 6.29 & 1.97 & 2.5 & 19.4 & 64 & 1825 & 0.66 & 7.27 & 1.76 & 1.7 & 21.4 & 43.9 \\
\hline 422 & 0.66 & 7.41 & 1.64 & 3.9 & 18.0 & 70 & 1866 & 0.66 & 7.11 & 1.78 & 4.2 & 20.9 & 55.9 \\
\hline 425 & 0.68 & 6.24 & 1.91 & 3.3 & 19.5 & 62 & 1879 & 0.67 & 8.26 & 2.49 & 4.0 & 40.7 & 35.9 \\
\hline 429 & 0.66 & 7.29 & 1.64 & 3.0 & 18.0 & 61 & 1922 & 0.67 & 7.22 & 1.79 & 0.9 & 22.0 & 39.3 \\
\hline 430 & 0.66 & 7.29 & 1.64 & 6.0 & 18.2 & 61 & 1924 & 0.66 & 7.01 & 1.83 & 2.0 & 24.5 & 39.7 \\
\hline 447 & 0.65 & 6.82 & 1.72 & 3.0 & 26.8 & 71 & 1930 & 0.67 & 7.17 & 1.80 & 0.5 & 20.9 & 36.9 \\
\hline 449 & 0.65 & 6.82 & 1.72 & 3.0 & 21.0 & 56 & 1942 & 0.68 & 6.85 & 1.77 & 0.9 & 21.3 & 49.2 \\
\hline 462 & 0.64 & 6.20 & 2.07 & 0.9 & 29.2 & 83 & & & & & & & \\
\hline 463 & 0.66 & 5.65 & 1.72 & 4.8 & 30.5 & 69 & & & & Average & & & \\
\hline 474 & 0.63 & 4.91 & 2.36 & 0.9 & 29.2 & 83 & & & & & & & \\
\hline 477 & 0.65 & 6.81 & 1.42 & 4.9 & 34.2 & 71 & & 0.66 & 7.07 & 1.96 & 3.07 & 23.82 & 60.60 \\
\hline 481 & 0.61 & 5.65 & 2.45 & 5.2 & 24.6 & 68 & & 0.00 & & 1.50 & (5. & & \\
\hline 482 & 0.65 & 5.71 & 1.89 & 1.0 & 31.0 & 151 & \multirow{2}{*}{\multicolumn{7}{|c|}{ Standard deviation }} \\
\hline 483 & 0.65 & 5.71 & 1.89 & 0.7 & 31.0 & 76 & & & & & & & \\
\hline $\begin{array}{r}485 \\
1630\end{array}$ & $\begin{array}{l}0.63 \\
0.67\end{array}$ & $\begin{array}{l}6.75 \\
8.08\end{array}$ & $\begin{array}{l}2.05 \\
2.29\end{array}$ & $\begin{array}{l}0.5 \\
3.2\end{array}$ & $\begin{array}{l}31.2 \\
26.3\end{array}$ & $\begin{array}{l}76 \\
43.2\end{array}$ & & 0.00 & 2.69 & 0.06 & 2.73 & 73.00 & 341.93 \\
\hline 1652 & 0.61 & 7.26 & 1.83 & 3.6 & 41.0 & 63.7 & & & & & & & \\
\hline 1670 & 0.66 & 7.24 & 1.76 & 2.7 & 18.9 & 54.4 & & & & $\mathrm{~A} / \mathrm{S}_{\mathrm{A}}$ & & & \\
\hline 1674 & 0.62 & 6.66 & 1.83 & 1.0 & 35.5 & 70.7 & & & & & & & \\
\hline 1685 & 0.65 & 7.25 & 1.76 & 2.0 & 23.8 & 57.6 & & -0.271 & 5.758 & 0.458 & 1.027 & 2.263 & 2.072 \\
\hline 1704 & 0.67 & 7.38 & 1.80 & 6.0 & 21.7 & 56.2 & & & & & & & \\
\hline 1712 & 0.67 & 7.13 & 1.74 & 1.2 & 21.3 & 56.5 & & & & $\mathrm{E} / \mathrm{S}_{\mathrm{E}}$ & & & \\
\hline 1730 & 0.69 & 7.25 & 2.04 & 4.2 & 16.8 & 52.6 & & & & & & & \\
\hline 1731 & 0.69 & 7.25 & 2.04 & 3.2 & 16.8 & 52.6 & & -0.385 & 39.74 & -0.687 & 3.003 & 8.981 & 7.666 \\
\hline 1732 & 0.70 & 7.35 & 2.03 & 3.0 & 16.6 & 49.6 & & & & & & & \\
\hline 1733 & 0.70 & 7.35 & 2.03 & 4.8 & 16.6 & 49.6 & & & & $\mathrm{~A} /(6 / \mathrm{N})^{0.5}$ & & & \\
\hline 1734 & 0.69 & 7.39 & 2.06 & 2.2 & 18.6 & 64.3 & & & & - & & & \\
\hline 1735 & 0.63 & 6.32 & 2.07 & 4.3 & 22.7 & 62.0 & & -0.890 & 18.952 & 1.506 & 3.380 & 7.449 & 6.819 \\
\hline 1747 & 0.66 & 7.25 & 1.76 & 10.0 & 22.2 & 53.3 & & -0.890 & 18.952 & 1.500 & 5.500 & 1.449 & 0.013 \\
\hline 1748 & 0.66 & 7.25 & 1.76 & 1.5 & 22.2 & 53.3 & & & & $\mathrm{E} /(24 / \mathrm{N})^{0.5}$ & & & \\
\hline 1756 & 0.67 & 7.53 & 2.37 & 2.5 & 25.5 & 37.0 & & & & & & & \\
\hline 1757 & 0.67 & 7.58 & 2.34 & 2.7 & 24.5 & 46.8 & & & & & & & \\
\hline 1757.5 & 0.67 & 7.58 & 2.34 & 2.0 & 24.5 & 46.8 & & 0.634 & 64.798 & -1.130 & 4.942 & 14.781 & 2.616 \\
\hline
\end{tabular}

Table 3 (continued).

\begin{tabular}{lrrrrrrr}
\hline & & \multicolumn{7}{c}{ Matrix of correlation } \\
\cline { 3 - 8 } & $\begin{array}{c}\text { Depth } \\
\text { (mbsf) }\end{array}$ & $\mathrm{Mg}^{*}$ & $\mathrm{CaO} / \mathrm{Na}_{2} \mathrm{O}$ & $\mathrm{Zr} / \mathrm{Y}$ & $\mathrm{Au}$ & $\mathrm{TiO}_{2} / \mathrm{Cr}$ & $\mathrm{Zn}$ \\
\hline & & & & & & & \\
& & & & & & & \\
$\mathrm{Depth}$ & 1.000 & 0.396 & 1.103 & -0.099 & -0.114 & -0.049 & -0.630 \\
$\mathrm{Mg}$ & 0.396 & 1.000 & -0.057 & 0.012 & -0.016 & -0.478 & -0.324 \\
$\mathrm{CaO} / \mathrm{Na}_{2}$ & 0.103 & -0.057 & 1.000 & -0.024 & 0.001 & -0.115 & -0.232 \\
$\mathrm{Zr} / \mathrm{Y}$ & -0.099 & 0.012 & -0.024 & 1.000 & 0.032 & 0.158 & 0.108 \\
$\mathrm{Au}$ & -0.114 & -0.016 & 0.001 & 0.032 & 1.00 & 0.030 & -0.102 \\
$\mathrm{TiO} / \mathrm{Cr}$ & -0.049 & -0.478 & -0.115 & 0.158 & 0.030 & 1.000 & 0.127 \\
$\mathrm{Zn}$ & -0.630 & -0.324 & -0.232 & 0.108 & -0.102 & 0.127 & 1.000 \\
\hline
\end{tabular}

